\author{
Military Technical College \\ Kobry El-Kobba \\ Cairo, Egypt
}

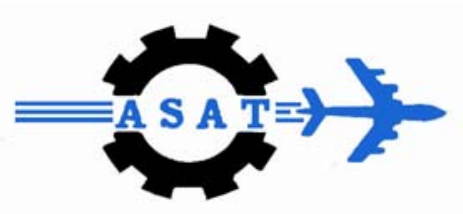

12-th International Conference

on

Aerospace Sciences \&

Aviation Technology

\title{
A NEW PATTERN FOR NUTATING RETICLES TO SIMPLIFY SEEKER ELECTRONICS
}

\author{
S. A. MORTAZAVI ${ }^{\star}$, A. MAHMOODI $^{\dagger}$ and M. R. ARVAN ${ }^{\ddagger}$
}

\begin{abstract}
Reticle systems have widely been used in IR seekers because of their simple structure and low cost; and they often employ stationary reticles with conical scan optics, for their extensive field of view (FOV) and high accuracy. The typical reticle pattern in these systems is the Wagon-Wheel reticle. This paper presents a new reticle pattern instead of Wagon-Wheel pattern in order to simplify the seeker electronics, while keeping the extended field of view. The proposed pattern requires AM demodulation instead of current FM demodulation. We have simulated target detection system by using the proposed reticle pattern. The results have confirmed our claim. The static gain curves have been obtained for different target sizes and positions for the proposed reticle and have been compared with that's of WagonWheel reticle.
\end{abstract}

\section{KEY WORDS}

IR Seeker- nutating system- Reticle pattern

\footnotetext{
MSc. Student, Dep. Of Physics, Applied Science Complex, Malek-e-Ashtar University of Technology, Shahinshahr, Iran.

† Assistant Professor, Physics Dep., Applied Science Complex, Malek-e-Ashtar University of Technology, Shahinshahr, Iran.

‡ Ph.D student, Dep. of Electrical Engineering, K. N. Toosi University of Technology, Tehran, Iran.
} 


\section{INTRODUCTION}

Infrared (IR) seekers use the heat produced by the target, that is typically an aircraft or helicopter, to locate and track it [1-6]. Vision systems of this seekers may be classified into imaging and non-imaging systems. Non-imaging systems, normally as reticle systems, are widely used in IR seekers. According to the type of relative motion produced between of reticle and target IR image, reticle seekers are classified into two types: rotating and stationary reticle seekers [1-3].

In the rotating reticle systems, the reticle pattern is rotationally moved in the image plane of the optical system. These systems have a significant limitation of loss of carrier signal for zero tracking error. By using the nutating reticle system, however, this limitation can be surmounted $[1,6]$.

In the nutating system, the image scene is moved over the stationary reticle. In order to scan the image in a circular path on the reticle, the nutating system employs the fixed-reticle and off-axis rotating lenses, canted mirrors, or wedged prisms. The center of the circular path corresponds to the target position in the field of view (FOV). Although the target is on the optical axis, the nutating system, on the contrary of rotating system, could produce the modulation signal because the circular path of target is concentric with that of the reticle [1-2].

Accuracy level in determination of the target position depends on various factors. The most significant of them include the light transmission pattern of reticle and detector output signal process algorithm. Up to now, various patterns have been proposed for the reticle, either stationary or rotating ones. Despite their particular limitations, each of the patterns offers some of the system requirements. However, the significant point is that it would be possible to get access to of design some patterns which are optimal for a system of the special specifications.

The signal demodulation from the Wagon-Wheel reticle system, witch is the most typical stationary reticle pattern, is at FM form. In this paper, we shall introduce a new stationary reticle pattern whose demodulation is at AM form. This pattern has a couple of considerable advantages. First, its signal demodulation algorithm is simpler than that of FM form; second, it would be more appropriate for tracking an extended field of view.

We are going to explain the demodulation procedure of the Wagon-Wheel reticle system and then we would like to propose the new reticle pattern as well as demodulation algorithm. Following that, the system will be simulated and the static gain curve in the extended field of view for the suggested reticle system will be obtained and compared with the Wagon-Wheel reticle system static gain curve. At the end, we are going to discuss the advantages and disadvantages points of the above mentioned reticle. 


\section{STATIONARY RETICLE SEEKER}

The Fig. 1 shows the scheme of a nutating reticle system. When the seeker and target are at null position, the conical scan causes to the target image rotate symmetrically around the center of the reticle pattern, producing a constant carrier frequency. This frequency is equal to the number of spoke pairs times the nutating frequency.

The typical simple of the stationary reticle is the Wagon-Wheel reticle. The reticle along with target image transmission paths are shown by the Fig. 2. The WagonWheel reticle includes $\mathrm{N}$ spoke pairs, and its radius corresponds with the field angle [7]. The nutating scan is used in this seeker in which a point source image rotates around a circle of constant radius $R_{n}$ and of nutating frequency $f_{n}$. The nutating circle center corresponds with the target position within a field of view, so that the nutating circles of target that located in the center of field of view will be concentric with the reticle.

When the target image passes through a transparent spoke, one pulse is produced, so that for the target in the center of field of view, width of the pulses and their distances are constant and equal. When the target getting away from the optical axis, width of pulses and their distances along the nutating circles will change, causing a modulated signal. The modulation will be just FM for the Wagon-Wheel reticle. The detector signal carrier frequency is $N \times f_{n}$ which have a frequency modulation with the sinusoidal signal of the frequency $f_{n}$, having amplitude in accordance with the tracking error amount. The modulation phase to the nutating phase reference equals a polar target angle.

The conical scan circle of the Wagon-Wheel reticle system is located almost in the middle of the reticle. For the on-axis target, the circle is concentric with the reticle and detector output will be a sinusoidal signal of the frequency $\mathrm{N} \times \mathrm{f}_{\mathrm{n}}$. As the target is getting away from the instantaneous field of view, the conical scan circle also moves, the output signal frequency will change proportionally to the target distance error during a period. The detector output signals, for the on and off-axis targets in the Wagon-Wheel system, are shown by the Fig. 3. In this system, radial and angular position of target will be obtained with high accuracy by means of the output signal frequency demodulation.

A normal algorithm for the FM signal demodulation is shown by the Fig. 4. Normally, at the beginning, the FM signal is passed through a bandpass filter at carrier frequency $f_{c}$, so that it will be converted to an AM-FM signal. This AM-FM signal has amplitude proportional to the FM signal frequency changes. The AM-FM signal will be passed through a rectifier and push detector, then a narrow bandpass filter of a central frequency $f_{n}$ is applied to it, and finally a clear demodulated signal is produced.

In the AM reticle system, the normal algorithm for the detector output signal demodulation includes blocks shown by Fig. 5 . First, the signal passes through a rectifier block and its amplitude becomes passive. Then, with the applying of a 
lowpass filter with cut-off frequency $f_{n}$ (nutation frequency or rotation period reverse) on it, the push will become obvious. For the signal to be clearer, a narrow bandpass filter with the central frequency $f_{n}$ will be applied on it. If the AM signal is passive before it enters the demodulation, like the reticle system output, there will be no need for the rectifier block.

As it has already been considered, in comparison to the AM demodulation, the FM demodulation has one more block; therefore its electronic process will be more complex. This benefit encourages us to the AM demodulators.

\section{NEW PROPOSED RETICLE PATTERN}

In case the AM demodulation is applied to the Wagon-Wheel reticle output, the resulted output will be null. The pulse amplitude constancy as well as local average constancy, as a result of lack of the components on $f_{n}$ within the frequency domain is among the reasons for the issue. For conversion of Wagon-Wheel reticle output signal from AM to FM, the spokes should be changed, so that the average output signal among within a nutation period stays constant. In other words, through this change, we intend to produce some components on $f_{n}$ in the frequency expand of the detector output signal.

In the Wagon-Wheel, when the target is at any position, its image in the nutation passes some intervals on the opaque and transparent spokes, accordingly the generated signal has no low-frequency changes. If these intervals are not equal, as the target image, in one period, is on the transparent spokes for less time, the signal symmetry will be disturbed.

In other words, for the AM to FM conversion of demodulation procedure of WagonWheel stationary reticle, there should be a kind of change that with the target image movement on the reticle pattern, passing intervals across the opaque and transparent spokes are not equal. Of course, it should be taken into account that for dependence of modulation depth on the target radial errors, for the on-axis target, the transmission intervals of opaque and transparent spokes should be equal and with more distance form the reticle center, further difference will be created among the interval. The reticle pattern, designed for this aim, is indicated by Fig. 6. This pattern due to its special design is called the Sunflower reticle.

The conical scan circle radius is equal to exactly half of the reticle radius, so that for the on-axis targets will be on the transparent spokes edge. The target image motion paths and generated signals for the targets with different positions are shown by the Fig. 7. As indicated, like the Wagon-Wheel reticle system, when the target is at the center of the field of view, intervals of the image passage through the black and white sectors of the reticle pattern are equal, and no modulation in the signal will be generated. It is expected that with further distance of the target from the tracking field center, in other words, transfer of the conical scan circle, some kind of the FM modulation is generated. In fact, this modulation is generated, but due to gradual thinning of the transparent spokes, turning on-off interval equality of the detector will be disturbed. This is why the signal partial average during a period changes. If these 
signals are passed through the lowpass filter with cut-off frequency $f_{n}$, the below mentioned signals indicated by the Fig. 8 will result.

Increasing in the signal amplitude by filtering according to the target radial error increase up to a point where the conical scan circle should not go out of the reticle, is approximately linear.

\section{SUNFLOWER RETICLE ADVANTAGES AND DISADVANTAGES}

The Sunflower reticle pattern has two significant advantages. First, as mentioned, for demodulation, fewer circuits will be required. Second, since when the conical scan circle goes out of the reticle, signal modulation and its demodulation is AM, without any requirement of switching and second circuit [7] in this reticle, with the same AM demodulator circuit, it is possible to generate the target position error signal.

The Fig. 9 shows the Sunflower reticle static gain for a target with radius $0.03 R_{r}$ and angular position zero, in the state of extended field of view. Like the Wagon-Wheel reticle, extended field of view radius in the reticle is $R_{r}+R_{n} . R_{r}$ and $R_{n}$ are the reticle radius and conical scan circle radius respectively.

Despite advantages of the reticle application instead of the Wagon-Wheel reticle, the Sunflower reticle pattern has four significant problems. First, is lack of strong pulse producing while the target image passes through the reticle center, producing the low error signals amplitude for targets with a radial error $R_{n}$ (Fig. 9(1)). The conical scan circle, generated signal by the detector and filtered signal for this state is shown by the Fig. 10-1 and 11-1. For removing this problem, it is possible to change the reticle pattern in the central areas. On the basis of it, two of the designed reticles will be indicated by the Fig. 13.

Due to the high modulation depth, the reticle pattern spokes (Fig. 13-a) are short. The more spoke width reduction rate for further distance from the edges, at the same extent rise in the error signal range velocity, and therefore, increase in the gain curve slope will result.

The second problem is passing of target image through distance among the transparent spokes for special target positions with radial error more than $R_{r}$. Several instances of these positions are shown by the Fig. 10-2. Some deep valleys in the static gain curve will be created as a result of the problem.

The third problem in this regard is error signal amplitude reduction for errors more than $R_{r}$, due to decrease in the detector output signal pulse number (Fig. 9(3)). The above-mentioned problem may be seen in all of the Stationary AM reticles.

The last problem regarding the Sunflower reticles is heterogeneous spatial filtering. The problem has no impact on the extracted static gain curve and will be important as one deal with such background as the shining and small clouds. In case the spatial reticle filtering is not heterogeneous, the shining backgrounds will add the sine 
components of conical system scan frequency to the detector output signal. In condition that the shining background is enough expanded and also reticle spatial filtering is uniform, its impact on the detector output signal will be an amount of DC in addition form. There are two reasons for the heterogeneous spatial filtering of the Sunflower reticle. First, is low average transmission of the external reticle ring, while its internal ring of transmission is equal to 0.5. The more transparent spokes width reduction rate is lower in respect of reticle radius increase, amount of average transmission of the ring will be closer to 0.5 , however this problem causes reduction in the modulation depth. When compensation of reduction in the modulation depth in the electronic demodulation is possible, it is likely to choose the spokes width reduction rate, so that the average transmission of the second ring will be closer to 0.5 .

The second reason, if the heterogeneous spatial filtering of the Sunflower reticle, which is true to the Wagon-Wheel reticle, is fluctuation of the spoke width and their distance in the entire pattern. For removing the problem, the winding pattern will be suitable [1].

\section{CONCLUSION}

All of the above-mentioned problems with the Sunflower reticle are true to the Wagon-Wheel reticle. Of course, about the two reticles, problems expect for the second problem may be somewhat removed by means of some corrections. However, in comparison to the Wagon-Wheel reticle, the Sunflower reticle enjoys two significant advantages. First, as mentioned, since the reticle pattern is AM, instead of $\mathrm{FM}$, in comparison to the Wagon-Wheel reticle system demodulation, its demodulation is simpler. Second, since stationary reticle system modulation for more than $R_{r}-R_{n}$, when the conical scan circle is not inside the reticle pattern, entirely, is AM $[1,6]$, without requirement of additional circuit, in the Sunflower reticle system, the error signal could be extracted. Moreover, there will be no proportionality any between the error signal in the area with the target position error level, but the target position phase would be accessible with high accuracy. By comparison, the extracted phase for both Sunflower reticle system and Wagon-Wheel reticle, which for the first one the AM demodulation and the second the FM demodulation, is indicated by the Fig. 14. As it can be seen, regarding the Wagon-Wheel reticle, unlike the Sunflower reticle, no correct phase for the radial errors more than $R_{r}-R_{n}$ has been obtained.

\section{References}

[1] Gerson G. And Rue A. K., "Tracking systems," Chap. 22 in The Infrared Handbook, Zissis G. J. and Wolfe W. L., Eds., ERIM, Ann Arbor, MI (1985).

[2] Hudson, R. C., "Infrared Systems Engineering" John Wiley \& Sons, New York (1969).

[3] Gustaf Olsson, "Simulation of reticle seekers by means of an image processing system," Optical Engineering, Vol.33, No. 3, pp. 730, March (1994).

[4] Lucien M. Biberman, "Reticles in Electro-Optical Devices," Chapter 3: Reticles for Error Signal Generators, pp. 25-42, Pergamon Press, New York, (1966). 
[5] Hyung-Ki Hong, Surng-Gahb Jahng, Kyoung-Soo Doo, and Jong-Soo Choi, "Reticles-Rotating Systems," Encyclopedia of Optical Engineering, pp. 2431, (2003).

[6] Jahng S.-G., Hong H.-K., Choi J.-S. and Han S.-H., "Reticles-Nutating Systems," Encyclopedia of Opt. Eng., pp. 2417-2430, (2003).

[7] Jeong-Su Oh, Kyung-Soo Doo, Surng-Gabb Jahng, Dong-Sun Seo, and JongSoo Choi, "Novel adaptive digital signal processing algorithm for a stationary reticle seeker," Optical Engineering, Vol. 39(10), pp. 2797-2803, Oct. 2000. 


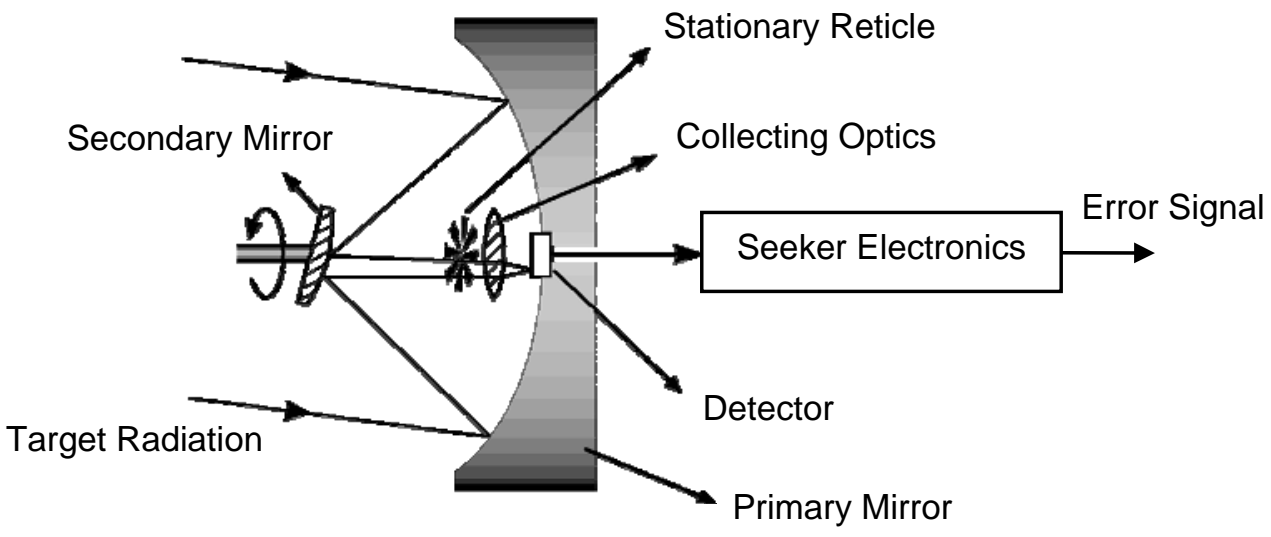

Fig. 1. The stationary reticle system

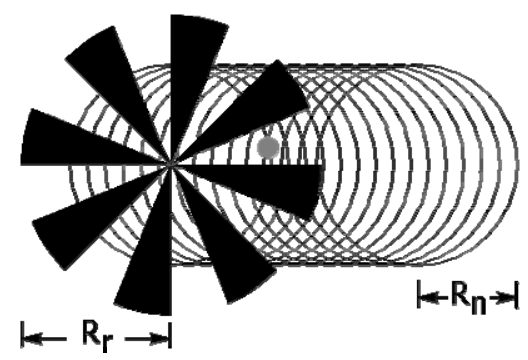

Fig. 2. Wagon-Wheel reticle and nutation circles of an IR target image
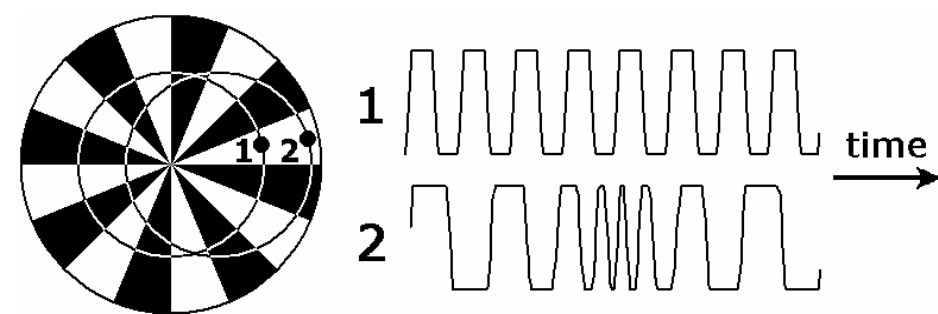

Fig. 3. Output signals for targets 1 . on-axis and 2. off-axis

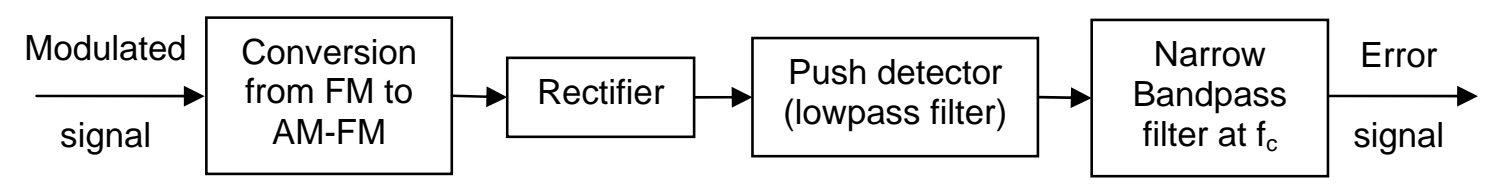

Fig. 4. Normal block diagram for FM demodulation

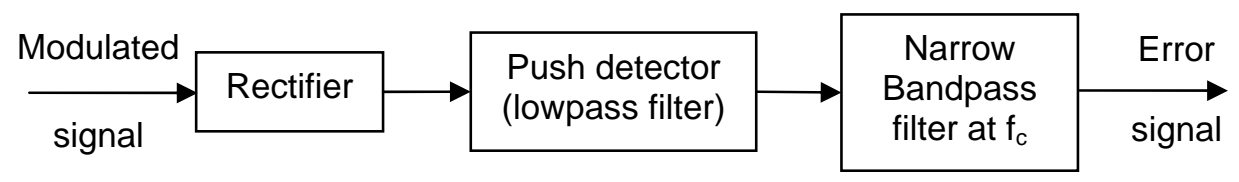

Fig. 5. Normal block diagram for AM demodulation 


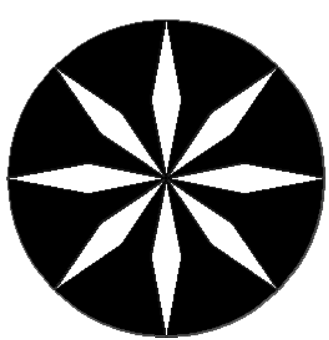

Fig. 6. The reticle pattern that has been designed to turn FM modulation into AM, called Sunflower reticle

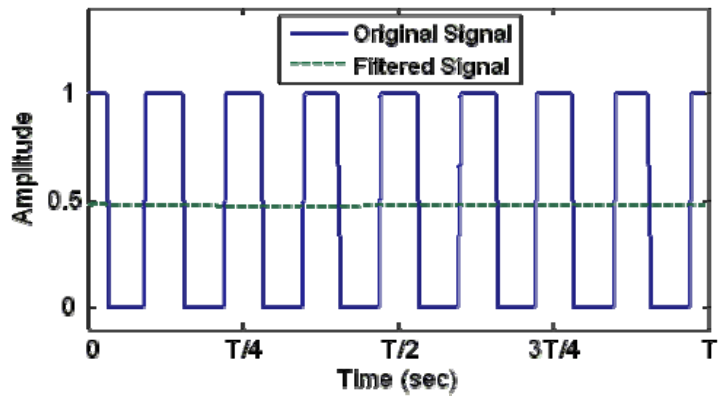

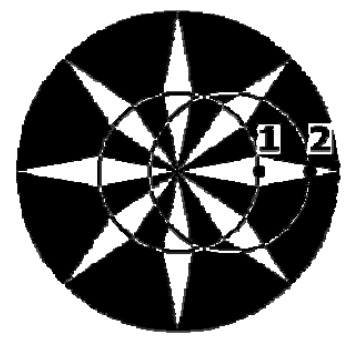

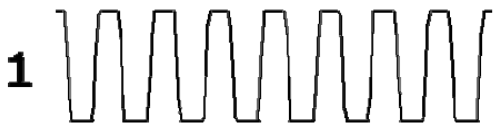

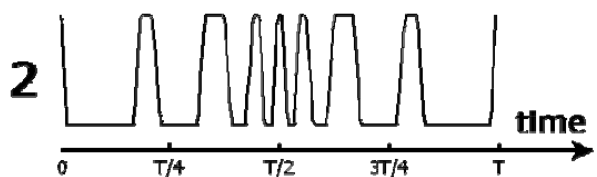

Fig. 7. Conical scan circles and generated signals by the Sunflower reticle for 1 . on-axis point target and 2. off-axis

Fig. 8. Lowpass filter results to signals of Fig. 7

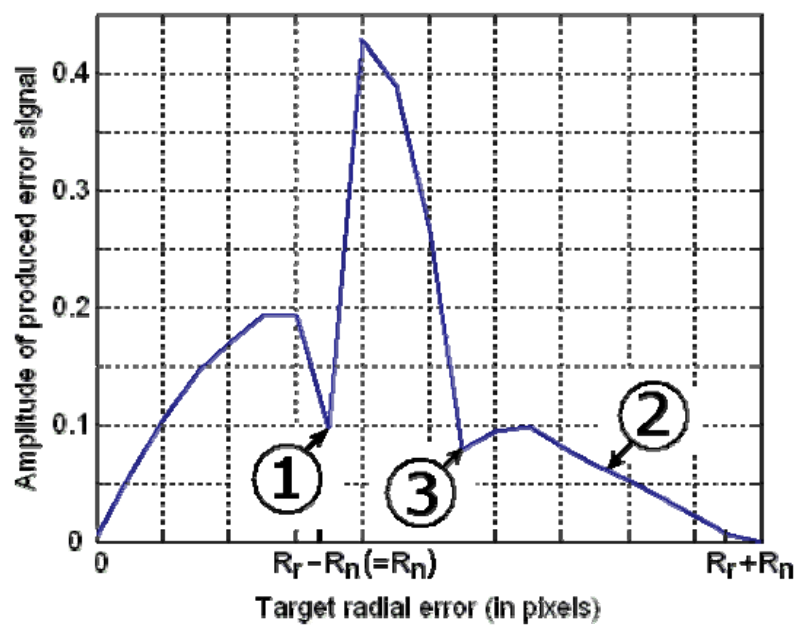

Fig. 9. Sunflower reticle static gain curve for extended field of view. The specific points with numbers are its main defects.
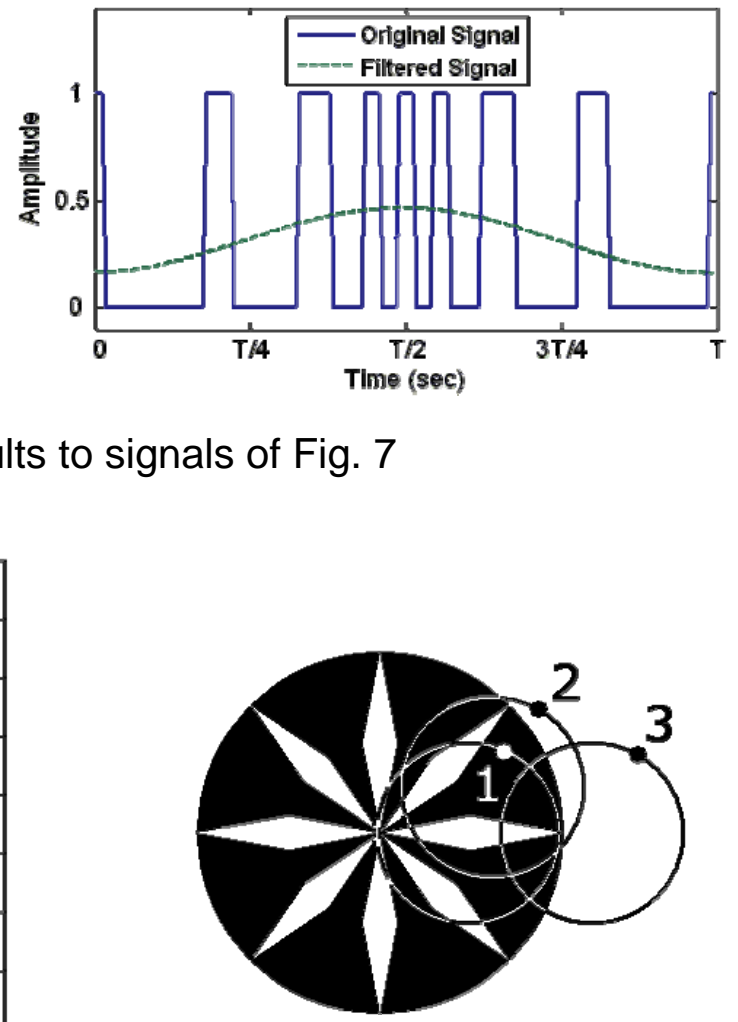

Fig. 10. Target positions which are reasons for incorrect error signals 

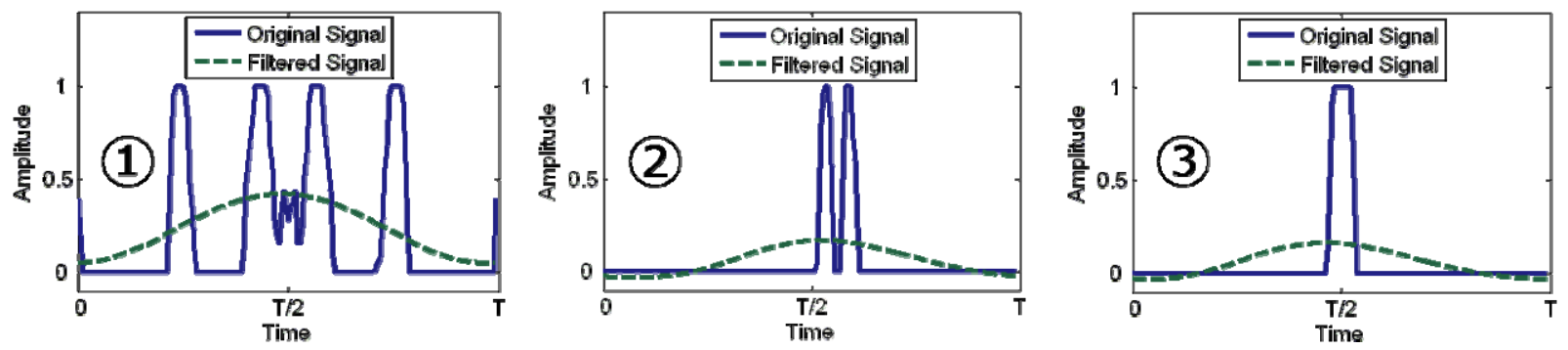

Fig. 11.
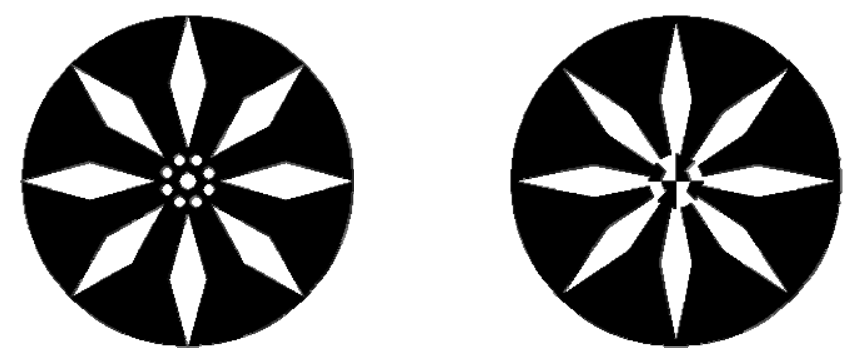

Fig. 12. Sunflower reticle with two central correction

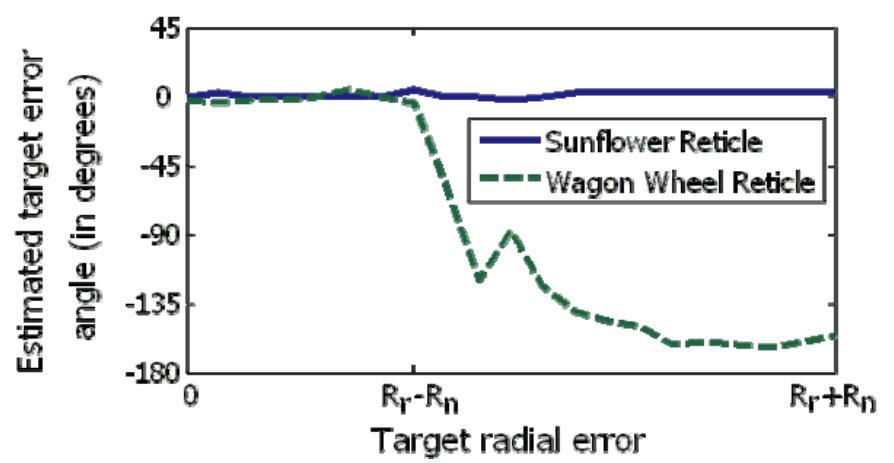

Fig. 13. Estimated target position phase for expanded FOV by using Sunflower and Wagon-Wheel reticles 